\title{
Neuroprotective effect of total flavonoids from Ilex pubescens against focal cerebral ischemia/reperfusion injury in rats
}

\author{
XIAOYAN FANG ${ }^{1}$, YUJIE LI $^{2}$, JINGYI QIAO ${ }^{3}$, YING GUO $^{4}$ and MINGSAN MIAO ${ }^{3}$ \\ ${ }^{1}$ Department of Pharmacology, School of Pharmacy; ${ }^{2}$ Pharmacology Laboratory, School of Basic Medicine; \\ ${ }^{3}$ Science and Technology Division, Henan University of Chinese Medicine, Zhengzhou, Henan 450046; \\ ${ }^{4}$ Institute of Bioengineering, Zhejiang Chinese Medical University, Hangzhou, Zhejiang 310053, P.R. China
}

Received November 2, 2016; Accepted June 26, 2017

DOI: $10.3892 / \mathrm{mmr} .2017 .7540$

\begin{abstract}
Ilex pubescens is commonly used in traditional Chinese medicine to treat cardiovascular and cerebrovascular diseases, such as coronary artery disease and stroke. However, the underlying mechanisms remain to be fully elucidated. The aim of the present study was to investigate the effects of Ilex pubescens total flavonoids (IPTF) on neuroprotection and the potential mechanisms in a rat model of focal cerebral ischemia/reperfusion (I/R) injury. Rats were pretreated with intragastric administration of IPTF at doses of 200 and $100 \mathrm{mg} / \mathrm{kg}$ for 5 days; middle cerebral artery occlusion surgery was then performed to induce cerebral I/R injury. Neurological deficits were determined using the 5-point neurological function score evaluation system, brain infarct sizes were determined by 2,3,5-triphenyltetrazolium chloride staining and alterations in brain histology were determined by hematoxylin and eosin staining. The neurological deficit score, the infarcted area and the brain tissue pathological injury were significantly reduced when the rats were pretreated with IPTF. In addition, inflammatory mediators and neurotrophic factors
\end{abstract}

Correspondence to: Professor Mingsan Miao, Science and Technology Division, Henan University of Chinese Medicine, 1 Jinshui East Road, Zhengzhou, Henan 450046, P.R. China

E-mail: miaomingsan@163.com

Abbreviations: I/R, ischemia/reperfusion; IPTF, Ilex pubescens total flavonoids; MCAO, middle cerebral artery occlusion; CCA, common carotid artery; ICA, internal carotid artery; TTC, 2,3,5-triphenyltetrazolium chloride; NOS, nitric oxide synthase; TNOS, total NOS; iNOS, induced NOS; cNOS, constitutive NOS; eNOS, endothelial constitutive NOS; nNOS, nervous constitutive NOS; NO, nitric oxide; IL-1 $\beta$, interleukin- $1 \beta$; TNF- $\alpha$, tumor necrosis factor- $\alpha$; BDNF, brain-derived neurotrophic factor; VEGF, vascular endothelial growth factor; GDNF, glial cell-derived neurotrophic factor; TCM, traditional Chinese medicine

Key words: Ilex pubescens total flavonoids, focal cerebral ischemia/reperfusion injury, neuroprotection, inflammatory cytokine, neurotrophic factor in the brain were investigated. IPTF pretreatment decreased the activities of total nitric oxide synthase (TNOS), induced NOS (iNOS) and constitutive NOS (cNOS), and the levels of nitric oxide (NO), interleukin- $1 \beta$ (IL-1 $\beta$ ) and tumor necrosis factor- $\alpha$ (TNF- $\alpha$ ), however, it increased the levels of IL-10 in brain tissues. Furthermore, pretreatment with IPTF also increased the protein expressions of brain-derived neurotrophic factor, glial cell-derived neurotrophic factor and vascular endothelial growth factor, when compared with the model group. In conclusion, the results of the present study demonstrated that IPTF has a neuroprotective effect against focal cerebral I/R injury in rats. The mechanism may be associated with the decreased production of certain proinflammatory cytokines including NO, IL- $1 \beta$, TNF- $\alpha$, TNOS, iNOS and cNOS, the increased production of the anti-inflammatory cytokine IL-10 and the increased secretion of neurotrophic factors.

\section{Introduction}

Stroke has become a leading cause of mortality and disability worldwide. In the USA and the UK, the morbidity of heart disease and cancer is reportedly $0.2 \%$ of the population each year. In China, 200 million people are affected by stroke every year, and as a result, $70-80 \%$ of these patients will suffer with life-changing disabilities (1). Strokes can be ischemic or hemorrhagic in nature; $~ 80 \%$ of strokes are ischemic stroke (2). Currently, therapy for ischemic stroke remains limited, and vascular recanalization and antiplatelet therapy are the only two therapeutic strategies that have been proven to be effective during the acute phase of ischemic stroke (3). For acute ischemic stroke, intravenous thrombolytic therapy within $4.5 \mathrm{~h}$ following the acute stroke is the most widely accepted method for vascular recanalization. but the narrow time window, hemorrhage complications and the low recanalization rates in patients with macrovascular occlusion often limit its use (4). Antiplatelet therapy is an important strategy for preventing recurrent stroke by simultaneously blocking different platelet activation pathways. Aspirin is the most widely used antiplatelet agent in current use, however, it has been reported that combination antiplatelet therapy with aspirin and clopidogrel increased the risk of hemorrhage complications $(5,6)$. Therefore, novel, effective and widely applicable pharmacological treatments are urgently required. 
The pathophysiology of stroke is very complex and involves a number of factors, including inflammation, oxidative stress, apoptosis and excitotoxicity (7). Ischemic stroke induces the expression of high levels of adhesion molecules, cytokines and inflammatory mediators, including nitric oxide (NO), interleukin-1 $\beta$ (IL-1 $\beta$ ) and tumor necrosis factor- $\alpha$ (TNF- $\alpha$ ). These high levels lead to marked local and systemic inflammatory responses $(8,9)$. Inflammation itself may exacerbate the spread of damage to the ischemic penumbra, increase the levels of inflammatory cytokines and thus, further aggravate brain injury (10). Therefore, the identification of novel drugs to regulate the inflammatory response is a promising method for the treatment of cerebral ischemic injury. Brain-derived neurotrophic factor (BDNF), a member of the neurotrophin family, serves an important role in the development and plasticity of the brain. It also protects the brain against injuries induced by stress conditions such as ischemia and trauma, by promoting nerve regeneration, preventing neuronal death, and regulating synaptic plasticity and cell survival (11). Glial cell-derived neurotrophic factor (GDNF) is another important neurotrophin, which serves an important role in reducing apoptosis in neurons and promoting neurological outcomes following hypoxia-ischemia, by inhibiting the production of caspase- $3, \mathrm{TNF}-\alpha$, NO and induced nitric oxide synthase (iNOS) (12). Vascular endothelial growth factor (VEGF) is an angiogenic peptide involved in nerve vascular remodeling, promoting growth in neurons and glial cells, and protecting neuronal tissues from cell death induced by hypoxia or ischemia in order to achieve neuroprotection (13). These observations have indicated that BDNF, GDNF and VEGF may be potential therapeutic agents for the prevention of neuronal cell death following cerebral ischemia.

Traditional Chinese medicine (TCM) is used due to its rich sources, low costs, few side effects and its multi-targeting effects $(14,15)$. Therefore, it is important to investigate the effects and mechanisms associated with anti-cerebral ischemia drugs used in TCM. The TCM Mao Dong Qing, the dry roots of Ilex pubescens Hook. et Arn, is an evergreen shrub that is predominantly found in the southern regions of China. It has been used primarily for the treatment of hypercholesterolemia and cardiovascular diseases, such as stroke and coronary artery disease, in addition to peripheral vascular disease (16-18). Preliminary studies by our group identified the beneficial effects of Ilex pubescens total flavonoids (IPTF) in rat and mice models of cerebral ischemia (19-21). However, its underlying mechanism and compound activity with regard to its effects against focal cerebral ischemia injury remain to be elucidated. In the present study, the effect of IPTF on a rat model of focal cerebral ischemia/reperfusion (I/R) injury, induced by middle cerebral artery occlusion (MCAO), was investigated. The potential mechanisms underlying the alterations in the levels of inflammation-associated molecules and the increased secretion of neurotrophic factors were also examined.

\section{Materials and methods}

Plantcollectionandidentification.Ilexpubescens was purchased from Henan Province Pharmaceutical Co., Ltd. (Zhengzhou, China) and was identified by Professor Chengming Dong and Professor Suiqing Chen from the Department of Medicinal
Plants, School of Pharmacy, Henan University of Chinese Medicine (Zhengzhou, China). The voucher specimen (no. XX20140916001) was kept in the group's laboratory.

Preparation of extracts. The total flavonoids were obtained from Ilex pubescens using the method described previously (22). Briefly, air-dried Ilex pubescens was treated twice with $70 \%$ aqueous ethanol $(1: 10, \mathrm{w} / \mathrm{v})$ for $1.5 \mathrm{~h}$, then a further $1 \mathrm{~h}$ under reflux conditions at $80^{\circ} \mathrm{C}$. The filtered extracts were concentrated in a vacuum evaporator at $60^{\circ} \mathrm{C}$ to obtain the crude extracts. The crude extracts were dissolved in water (adjusted to $\mathrm{pH} 4.5$ ), and loaded into glass columns, which were wet-packed with AB-8 macroporous resin. Following elution with water and $10 \%$ aqueous ethanol, the elutriant with $40 \%$ ethanol was collected, dried using a vacuum at $60^{\circ} \mathrm{C}$ and then stored at $4^{\circ} \mathrm{C}$. The total flavonoid content in the extract was $>50 \%$, as determined by ultraviolet spectrophotometry, performed as described in a previous study (23).

Animal treatment and administration. A total of 96 adult male Wistar rats [SPF grade; age, 8 weeks; weight, 280-300 g; Laboratory Animal Center of Hebei Province, Shijiazhuang, China (certificate no. 907048)], were employed in the present study. Rats were housed in an environmentally controlled breeding room $\left(22-24^{\circ} \mathrm{C} ; 50-55 \%\right.$; 12 -h dark/light cycle), with free access to standard laboratory food and water. The present study was approved by the Ethics Committee of Henan University of Chinese Medicine (Henan, China). Rats were divided into the following 4 groups ( $n=24 /$ group): Sham operation group [sham; intragastric (ig) administration of saline and surgery without MCAO], ischemic group (model group; MCAO surgery with ig administration of saline), and the third and fourth groups were pretreated with a high (200 mg/kg/day; IPTF-1 group) or low ig dose of IPTF (100 mg/kg/day; IPTF-2) for 5 days, followed by the induction of I/R (MCAO surgery + IPTF pretreatment groups).

Induction of focal cerebral I/R via MCAO surgery. An hour following the last administration of IPTF pretreatment, all rats were anesthetized with chloral hydrate $(300 \mathrm{mg} / \mathrm{kg}$, intraperitoneally; Tianjin Zhiyuan Chemical Reagent Co., Ltd., Tianjin, China) and MCAO surgery was then performed according to Koizumi et al (24) and Nagasawa and Kogure (25) with a few modifications. Briefly, the left common carotid artery (CCA) and internal carotid artery (ICA) were isolated via a cervical midline incision. A nylon monofilament pre-coated with silicone rubber (0.26 mm external diameter) was inserted into the CCA and advanced into the ICA to $\sim 19-20 \mathrm{~mm}$ from the left CCA bifurcation to occlude the middle cerebral artery. Then, $2 \mathrm{~h}$ after the induction of ischemia, the filament was slowly withdrawn and animals were returned to their cages for $22 \mathrm{~h}$ of reperfusion. Rats in the sham operation group received the same surgical procedures without filament insertion to induce MCAO. Rat body temperature was maintained at $37.0^{\circ} \mathrm{C}$ using an infrared lamp throughout surgical procedures. After the surgery, 22 rats in the sham group, 20 rats in the model group, 20 rats in the IPTF-1 group and 22 rats in the IPTF-2 group survived, respectively.

Neurological deficit scores. Neurological deficit scores were recorded for each rat $22 \mathrm{~h}$ after ischemic injury 
( $\mathrm{n}=10-11$ rats/group) in a blind-controlled manner, according to Longa's five-point scale method (26). The following grading system was applied: Grade 0, no deficit; grade 1, failure to extend right forepaw; grade 2, circling to right; grade 3, falling to right; grade 4 , inability to walk spontaneously and a reduced level of consciousness.

Cerebral infarct volume measurement. Following behavioral testing, rats ( $n=10-11$ rats/group) were anesthetized with chloral hydrate $(300 \mathrm{mg} / \mathrm{kg}$, intraperitoneally) and sacrificed, and the brain tissues removed and separated into 6 coronal slices of 2-mm thickness. Brain slices were stained with $1 \%$ 2,3,5-triphenyltetrazolium chloride (TTC; cat. no. T8877; Sigma-Aldrich; Merck KGaA, Darmstadt, Germany) solution in the dark at $37^{\circ} \mathrm{C}$ for $15 \mathrm{~min}$. The cerebral infarct volume was measured using the method described by Belayev et al (27). In order to eliminate the contribution of swelling/edema to the ischemic lesion, the cerebral infarct volume was calculated with the following formula: Corrected infarct volume $=$ contralateral hemisphere volume $-($ ipsilateral hemisphere volume - measured infarct volume) (28).

Tissue preparation. The remaining rats ( $\mathrm{n}=10-11 /$ group) were anesthetized with $10 \%$ chloral hydrate $(300 \mathrm{mg} / \mathrm{kg}$ intraperitoneally), which was followed by rapid removal of the brain. The olfactory bulbs were removed and the brains were cut coronally into two parts at the optic nerve intersection. The first part of the tissue beginning at the rostral end was used to produce a $10 \%$ brain homogenate with saline, followed by centrifugation at $2,300 \mathrm{x}$ for $15 \mathrm{~min}$ at $4^{\circ} \mathrm{C}$. The supernatant was collected and stored at $-20^{\circ} \mathrm{C}$ until assay experiments. The remaining tissue was post-fixed in $4 \%$ paraformaldehyde (cat. no. AR1068; Wuhan Boster Biological Technology Co., Ltd., Wuhan, China) in $0.1 \mathrm{~mol} / 1$ phosphate-buffered saline (PBS; pH 7.4; cat. no. P1010; Beijing Solarbio Science \& Technology Co., Ltd., Beijing, China) at $4^{\circ} \mathrm{C}$ for 1 week for histological and immunohistochemical examination.

Detection of the levels of $I L-1 b, T N F-\alpha$ and $I L-10$ in brain homogenate. The supernatant of the brain homogenate was used to detect the levels of IL-1 $\beta$ (cat. no. SXM026), TNF- $\alpha$ (cat. no. SX01165) and IL-10 (cat. no. SXB005) using ELISA kits (all from Shanghai Senxiong Biotech Industry Co., Ltd., Shanghai, China) according to the manufacturer's protocols.

Detection of the content of NO and the activities of total NOS (TNOS) and iNOS in brain homogenate. The content of NO in brain tissue homogenate was assayed using the nitrate reductase method and an NO assay kit (cat. no. A012; Nanjing Jiancheng Bioengineering Institute, Nanjing, China) according to the manufacturer's instructions. The level was expressed as $\mu \mathrm{mol} / \mathrm{g}$ protein. The activities of TNOS and iNOS were measured using TNOS and iNOS assay kits (NOS kit, cat. no. A014-1; TP kit, cat. no. A045-2; Nanjing Jiancheng Bioengineering Institute, Nanjing, China) according to the manufacturer's instructions; results are expressed as $\mathrm{U} / \mathrm{mg}$ protein.

Hematoxylin and eosin $(H \& E)$ staining. A total of 6 fixed brain tissues were randomly selected from each group, dehydrated with graded alcohol series (80, 95 and 100\%) and embedded in paraffin. Then, sliced into coronal 5- $\mu \mathrm{m}$-thick sections, at least 6 from every sample. Then standard H\&E staining was performed according to $\mathrm{H} \& \mathrm{E}$ staining kit (cat. no. G1120; Beijing Solarbio Science \& Technology Co., Ltd.). Briefly, the sections were dewaxed with dimethylbenzene (Yantai Shuangshuang Chemical Co., Ltd., Yantai, China), and the slices were rehydrated with graded alcohol series $(100,95$, 80 and $70 \%$ ) for $5 \mathrm{~min}$ in each concentration and washed with running water twice in turn. Then, the slices were immersed in Harris hematoxylin for $5 \mathrm{~min}$, differentiated for $0.5 \mathrm{~min}$, and immersed in tap water for $15 \mathrm{~min}$ to return the nuclei to blue. Next, the slices are immersed in $70-80 \%$ alcohol for $1 \mathrm{~min}$ and in $0.5 \%$ eosin solution for 2 min. dehydrated with graded alcohol series (95 and 100\%) for $2 \mathrm{~min}$ in each concentration, cleared in dimethylbenzene and sealed with neutral balsam. All of the steps above were carried out at room temperature. Neuronal death in the cortical subfield of the ischemia side was evaluated under a light microscope in 2 high power microscopic fields (magnification, x400; Olympus BX50; Olympus Corporation, Tokyo, Japan). They were then scored with the following histological grading system (29): I, a few neurons damaged; II, numerous neurons damaged; III, majority of neurons damaged; IV, vast majority of neurons damaged.

Immunohistochemical staining. The remaining paraffinembedded sections were used to detect the expressions of BDNF, GDNF and VEGF. Briefly, 3 sections were deparaffinized, rehydrated with a graded alcohol series and washed with PBS. Then the sections were incubated in $3 \% \mathrm{H}_{2} \mathrm{O}_{2}$ at room temperature for $10 \mathrm{~min}$ to quench the endogenous peroxidase activity and rinsed in PBS. Following these procedures, an antigen retrieval procedure was performed by treating the samples in $0.01 \mathrm{M}$ sodium citrate buffer solution ( $\mathrm{pH} 6.0$, cat. no. C1120; Beijing Solarbio Science \& Technology Co., Ltd.) at $98-100^{\circ} \mathrm{C}$ for $20 \mathrm{~min}$. Samples were then subjected to incubation in $5 \%$ normal goat serum (Wuhan Boster Biological Technology Co., Ltd.) in $0.01 \mathrm{M}$ PBS for $30 \mathrm{~min}$ at room temperature. Sections were incubated with rabbit polyclonal anti-BDNF, anti-GDNF or anti-VEGF antibodies (anti-BDNF; cat. no. BA0565-1; anti-GDNF; cat. no. BA0890 and anti-VEGF, cat. no. BA0407; all 1:50 and from Wuhan Boster Biological Technology Co., Ltd.), respectively, in PBS for $2 \mathrm{~h}$ at $37^{\circ} \mathrm{C}$. The negative control sections were incubated with $5 \%$ normal goat serum for $2 \mathrm{~h}$ at $37^{\circ} \mathrm{C}$. Sections were then incubated with biotin-labeled secondary antibody (polyclonal goat anti-rabbit IgG/streptavidin antibody; 1:50; OriGene Technologies, Inc., Beijing, China) at $37^{\circ} \mathrm{C}$ for $30 \mathrm{~min}$, and then incubated with a Strept-Avidin-Biotin-Peroxidase complex (SABC-POD; cat. no. SA1025; Wuhan Boster Biological Technology Co., Ltd.) for $30 \mathrm{~min}$ at $37^{\circ} \mathrm{C}$. The results were visualized using a diaminobenzidine kit (DAB, cat. no. AR1022, Wuhan Boster Biological Technology Co., Ltd.). Finally, the sections were mounted onto polylysine-coated slides and all slides were photographed under a light microscope (magnification, x400; Olympus BX50). The intensity of BDNF, GDNF and VEGF in the cortex and hippocampus CA1 in each section was evaluated using Image-Pro Plus, version 5.1 (Media Cybernetics, Inc., Rockville, MD, USA) in two high power microscopic fields, and expressed as the positive signal area and the integrated optical density. 
Table I. Effect of IPTF on the neurological deficit scores of rats following MCAO.

\begin{tabular}{lll}
\hline Group & $\mathrm{n}$ & \multicolumn{1}{c}{ NDS } \\
\hline Sham & 11 & $0.00 \pm 0.00^{\mathrm{a}}$ \\
Model & 10 & $2.50 \pm 0.71$ \\
IPTF-1 & 10 & $1.80 \pm 0.63^{\mathrm{b}}$ \\
IPTF-2 & 11 & $2.00 \pm 0.79$ \\
\hline
\end{tabular}

Values are expressed as the mean \pm standard deviation. The following scoring system was used: 0 , no deficit; 1 , failure to extend right forepaw; 2 , circling to right; 3 , falling to right; 4 , inability to walk spontaneously and a reduced level of consciousness. ${ }^{a} \mathrm{P}<0.01$ and ${ }^{\mathrm{b}} \mathrm{P}<0.05$ vs. model group. IPTF, Ilex pubescens total flavonoids; NDS, neurological deficit score; model, middle cerebral artery occlusion without pretreatment; IPTF-1, rats pretreated with $200 \mathrm{mg} / \mathrm{kg}$ IPTF; IPTF-2, rats pretreated with $100 \mathrm{mg} / \mathrm{kg}$ IPTF.

Statistical analysis. All statistical analyses were performed using SPSS software, version 13.0 (SPSS, Inc., Chicago, IL, USA). Enumeration data were analyzed using the Kruskal-Wallis test, and the Mann-Whitney U-test was conducted for comparisons between groups. Measurement data were analyzed by a one-way analysis of variance and a least significant difference post hoc test was used for comparisons between groups. Data are presented as the mean \pm standard deviation. $\mathrm{P}<0.05$ was considered to indicate a statistically significant difference.

\section{Results}

IPTF treatment reduces brain damage in cerebral ischemic rats induced by MCAO. Neurological deficit scores, cerebral infarct volume and brain histopathology were investigated to determine the neuroprotective effects of IPTF in cerebral ischemic rats induced by MCAO. The results of the neurological deficit scores are displayed in Table I. The neurological deficit score of the model group was $2.50 \pm 0.71$ and when compared with the sham operation group there was a significant difference $(\mathrm{P}<0.01)$. Also, when compared with the model group, pretreatment with $200 \mathrm{mg} / \mathrm{kg}$ IPTF significantly reduced the impairment in neurological function induced by $I / R$ (IPTF-1 group; neurological deficit score, $1.80 \pm 0.63$; $\mathrm{P}<0.05$ ). The results indicated that IPTF pretreatment may improve behavioral injury in rats following MCAO.

The cerebral infarct volume was determined by TTC staining. The infarcted tissue was stained white and normal tissues were red. When compared with the model group, IPTF pretreatment (200 and $100 \mathrm{mg} / \mathrm{kg}$ ) significantly reduced the infarct volume induced by MCAO (Fig. 1A and B).

The results of $\mathrm{H} \& \mathrm{E}$ staining revealed that the cell membrane and nucleus of neurons in the cerebral cortex subfield were clearly visible in the sham operation group. When compared with the sham operation group, marked pathological lesions were visible in the $\mathrm{I} / \mathrm{R}$ model group. Neurons that were swollen, atrophic, lightly stained and undergoing necrosis were observed in the $\mathrm{I} / \mathrm{R}$ model group. Pathological lesions induced by cerebral ischemia were markedly reduced when compared
A
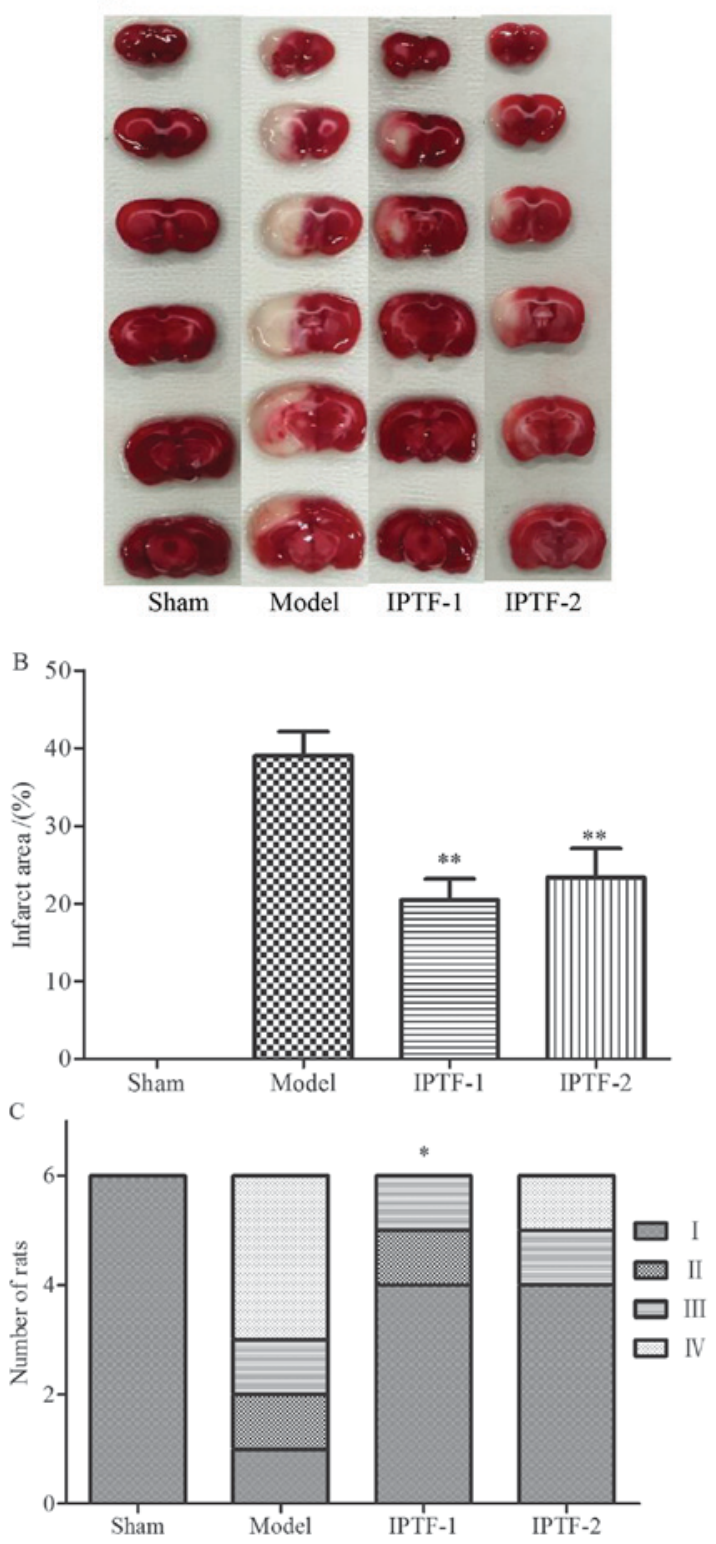

Figure 1. Effect of IPTF on cerebral infarction and the histological grades in the cortex of MCAO model rats. (A) Representative 2,3,5-triphenyltetrazolium chloride-stained brain coronal sections obtained from the sham operated, model (MCAO operated), IPTF-1 (200 mg/kg pretreatment) and IPTF-2 (100 mg/kg pretreatment) groups. The normal tissue was stained red while the infarct area was white. (B) v rate identified in the four groups. Results were quantified using ImageJ software and values are expressed as the mean \pm standard deviation. (C) Neuronal death in the cortical subfield of ischemia side was evaluated under a light microscope and scored by histological grade ( $\mathrm{n}=6$ random rat brain tissues/group). Data were analyzed using the Kruskal-Wallis test. The number of rat tissues exhibiting each histological grade is presented. The following histological grading system was used: I, a few neurons damaged; II, numerous neurons damaged; III, majority of neurons damaged; IV, vast majority of neurons damaged. ${ }^{*} \mathrm{P}<0.05$ and ${ }^{* *} \mathrm{P}<0.01$ vs. model group. IPTF, Ilex pubescens total flavonoids; MCAO, middle cerebral artery occlusion; IPTF-1, rats pretreated with $200 \mathrm{mg} / \mathrm{kg}$ IPTF; IPTF-2, rats pretreated with $100 \mathrm{mg} / \mathrm{kg}$ IPTF.

with the model group in the IPTF pretreatment groups. The results of the corresponding histological grading supported the histological results, the number of neurons with edema, that were atrophic or necrotic, were reduced in the IPTF pretreatment groups, particularly in the high-dose IPTF group (IPTF-1; P<0.05; Figs. 1C and 2). These results illustrated that 
Table II. Effect of IPTF on the content of IL-1 $\beta$, TNF- $\alpha$ and IL-10 in the brain tissue of rats with middle cerebral artery occlusion.

\begin{tabular}{lrccr}
\hline Group & $\mathrm{n}$ & $\mathrm{IL}-1 \beta(\mathrm{ng} / \mathrm{g}$ prot $)$ & TNF- $\alpha$ (ng/g prot) & IL-10 (ng/g prot) \\
\hline Sham & 10 & $9.83 \pm 2.76^{\mathrm{a}}$ & $2.00 \pm 0.95^{\mathrm{b}}$ & $9.34 \pm 4.06$ \\
Model & 9 & $13.01 \pm 2.86$ & $4.24 \pm 1.61$ & $12.00 \pm 3.24$ \\
IPTF-1 & 9 & $9.28 \pm 3.89^{\mathrm{a}}$ & $2.85 \pm 1.19^{\mathrm{a}}$ & $19.75 \pm 10.07^{\mathrm{a}}$ \\
IPTF-2 & 9 & $11.85 \pm 3.34$ & $2.84 \pm 1.54^{\mathrm{a}}$ & $18.08 \pm 10.38$ \\
\hline
\end{tabular}

Values are expressed as the mean \pm standard deviation. ${ }^{a} \mathrm{P}<0.05$ and ${ }^{\mathrm{b}} \mathrm{P}<0.01$ vs. model group. IPTF, Ilex pubescens total flavonoids; IL, interleukin; TNF- $\alpha$, tumor necrosis factor- $\alpha$; model, middle cerebral artery occlusion without pretreatment; IPTF-1, rats pretreated with $200 \mathrm{mg} / \mathrm{kg}$ IPTF; IPTF-2, rats pretreated with $100 \mathrm{mg} / \mathrm{kg}$ IPTF.

Table III. Effect of IPTF on the activities of total-, induced- and constitutive nitric oxide synthase, and the content of nitric oxide in brain tissues of rats with middle cerebral artery occlusion.

\begin{tabular}{llcccc}
\hline Group & $\mathrm{n}$ & TNOS (U/mg prot) & iNOS (U/mg prot) & cNOS (U/mg prot) & NO $(\mu$ mol/g prot) \\
\hline Sham & 11 & $1.86 \pm 0.79^{\mathrm{a}}$ & $0.49 \pm 0.23^{\mathrm{a}}$ & $1.02 \pm 0.32^{\mathrm{a}}$ & $5.64 \pm 6.11^{\mathrm{a}}$ \\
Model & 10 & $2.85 \pm 0.53$ & $0.89 \pm 0.26$ & $2.04 \pm 0.59$ & $14.96 \pm 5.26$ \\
IPTF-1 & 10 & $1.58 \pm 0.70^{\mathrm{a}}$ & $0.50 \pm 0.17^{\mathrm{a}}$ & $0.90 \pm 0.48^{\mathrm{a}}$ & $10.27 \pm 4.00^{\mathrm{b}}$ \\
IPTF-2 & 11 & $1.48 \pm 0.40^{\mathrm{a}}$ & $0.41 \pm 0.19^{\mathrm{a}}$ & $1.08 \pm 0.43^{\mathrm{a}}$ & $9.57 \pm 4.32$ \\
\hline
\end{tabular}

Values are expressed as the mean \pm standard deviation. ${ }^{a} \mathrm{P}<0.01$ and ${ }^{\mathrm{b}} \mathrm{P}<0.05$ vs. model group. IPTF, Ilex pubescens total flavonoids; NOS, nitric oxide synthase; TNOS, total NOS; iNOS, induced NOS, cNOS, constitutive NOS; NO, nitric oxide; model, middle cerebral artery occlusion without pretreatment; IPTF-1, rats pretreated with $200 \mathrm{mg} / \mathrm{kg}$ IPTF; IPTF-2, rats pretreated with $100 \mathrm{mg} / \mathrm{kg}$ IPTF.

pretreatment with IPTF may reduce brain injury induced by $\mathrm{MCAO}$ in rats.

Effects of IPTF on the inflammatory response in cerebral ischemic injury. The levels of IL-1 $\beta$, IL-10 NO and TNF- $\alpha$ in rat brains were detected in order to determine the influence of IPTF on the inflammatory response in cerebral $\mathrm{I} / \mathrm{R}$ injury. The results are presented in Table II. The levels of IL- $1 \beta$ and TNF- $\alpha$ in the ischemic hemispheres of the model group were significantly increased when compared with the sham operation group $(\mathrm{P}<0.05$ and $\mathrm{P}<0.01$, respectively). The level of IL-1 $\beta$ in the IPTF-1 treatment group $(200 \mathrm{mg} / \mathrm{kg}$ IPTF) was significantly decreased when compared with the model group $(\mathrm{P}<0.05)$. In addition, the levels of TNF- $\alpha$ were significantly decreased in the two IPTF pretreatment groups $(\mathrm{P}<0.05)$ when compared with the model group. By contrast, the expression of IL-10 in the model group exhibited no significant change when compared with the sham operation group. The $100 \mathrm{mg} / \mathrm{kg}$ IPTF pretreatment group had a tendency to increased expression of IL-10, but no significant difference $(\mathrm{P}>0.05)$ was observed when compared with the model group. However, it was significantly increased in the $200 \mathrm{mg} / \mathrm{kg}$ IPTF treatment group (IPTF-1; $\mathrm{P}<0.05$; Table II).

$\mathrm{NO}$, as a pre-inflammatory mediator, is derived from NOS and serves an important role in cerebral ischemia and the effects of an ischemic insult (30). Table III indicates that the levels of TNOS, iNOS, constitutive NOS (cNOS) and NO in the ischemic hemispheres were significantly increased in model group when compared with the sham operation group. In addition, IPTF pretreatment (100 and $200 \mathrm{mg} / \mathrm{kg}$ )
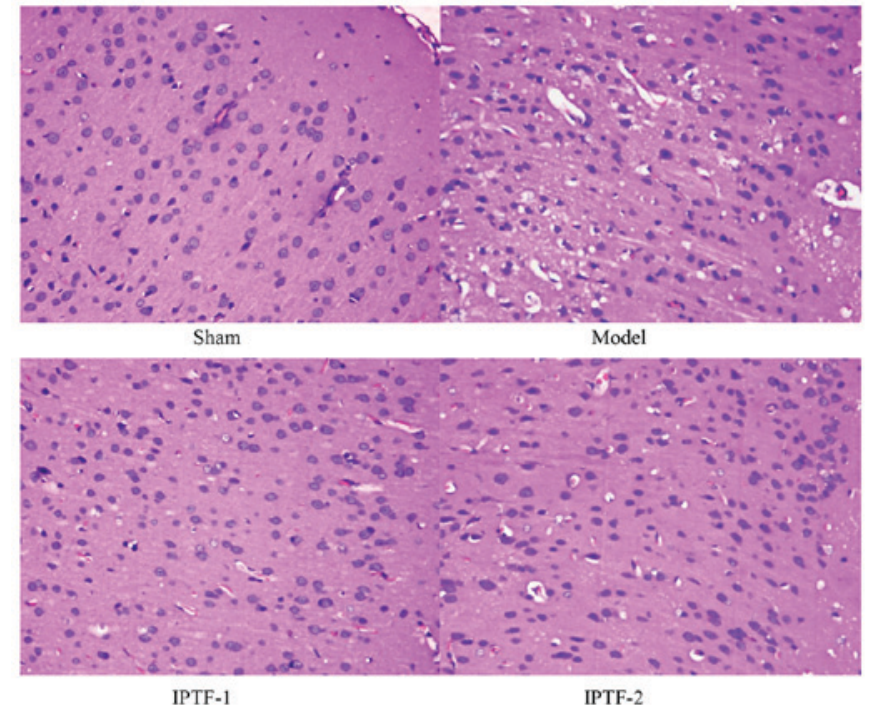

Figure 2. Effect of IPTF on the histopathological changes in the cortex of rats following MCAO. Representative images of hematoxylin and eosin stained tissues are presented (magnification, x400). IPTF, Ilex pubescens total flavonoids; MCAO, middle cerebral artery occlusion; model, MCAO operated without pretreatment; IPTF-1, rats pretreated with $200 \mathrm{mg} / \mathrm{kg}$ IPTF; IPTF-2, rats pretreated with $100 \mathrm{mg} / \mathrm{kg}$ IPTF.

significantly downregulated the levels of TNOS, iNOS, cNOS and NO in the ischemic hemispheres of rats when compared with the model group $(\mathrm{P}<0.05$ and $\mathrm{P}<0.01$; Table III).

In conclusion, the results demonstrated that IPTF reduced the inflammatory response by regulating the levels of IL-1 $\beta$, 

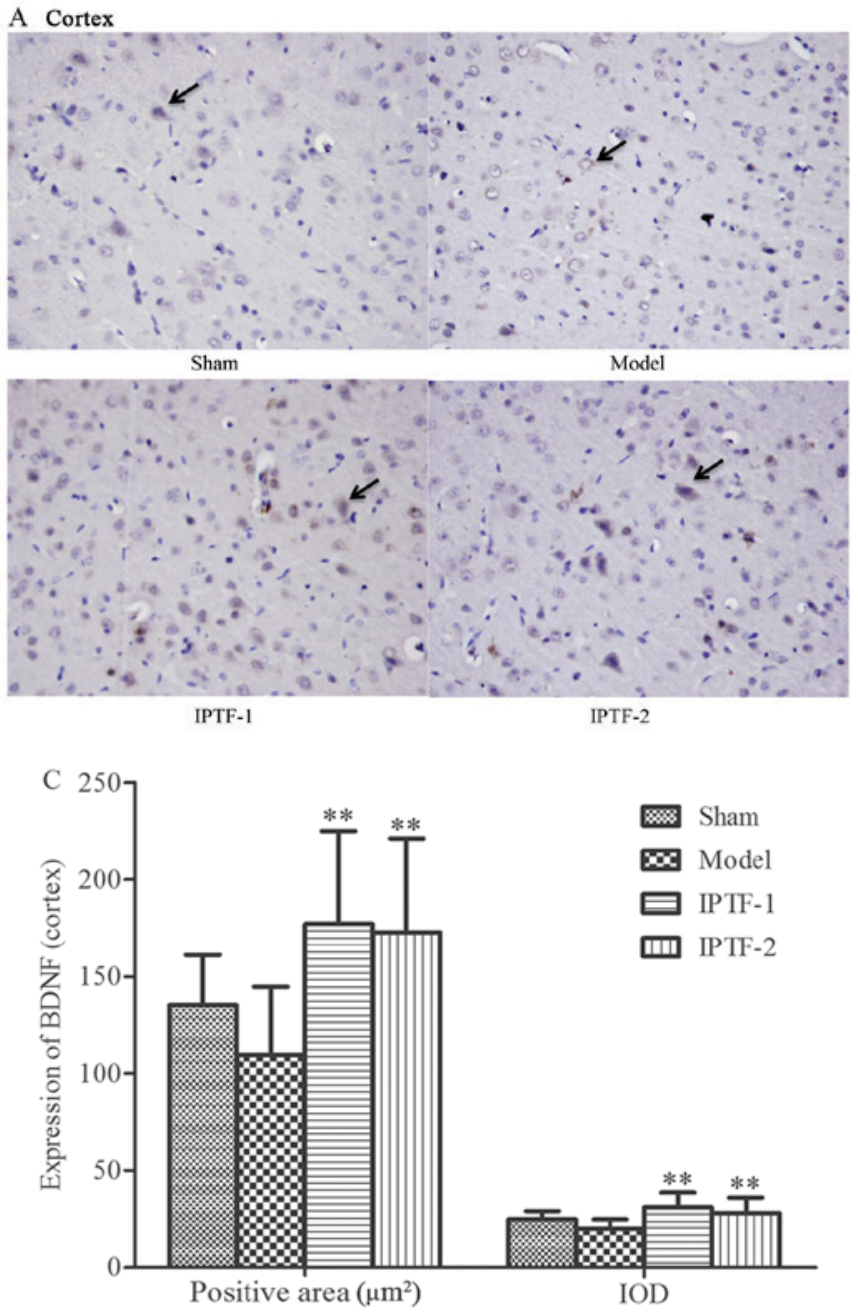

B Hippocampus
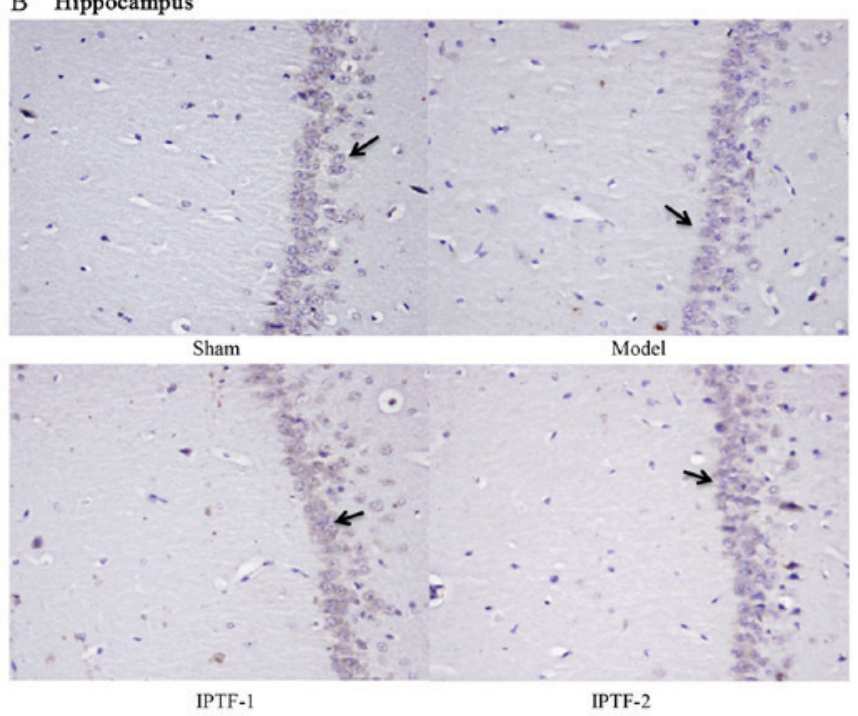

D

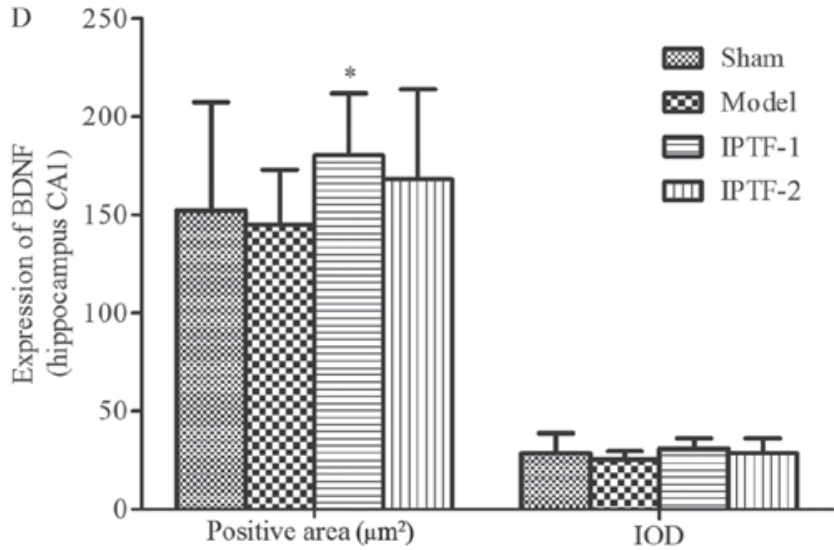

Figure 3. Effect of IPTF on the expression of BDNF in the cortex and hippocampus CA1 subfields of MCAO rats. Protein expression levels of BDNF were detected by immunohistochemical staining in (A) the cortex and (B) in hippocampus CA1 subfields. BDNF is stained brown and indicated by arrows; it was primarily localized in the cytoplasm of neurons. Magnification, $\mathrm{x} 400$. BDNF-positive immunohistochemical products in (C) the cortex and (D) hippocampus CA1 subfields were counted using the Image-Pro Plus 5.1 system, and are expressed as the positive signal area ( $\left.\mu \mathrm{m}^{2}\right)$ and the IOD. Values are expressed as the mean \pm standard deviation ( $\mathrm{n}=6$ random rat brain tissues/group). ${ }^{*} \mathrm{P}<0.05$ and ${ }^{* *} \mathrm{P}<0.01$ vs. model group. IPTF, Ilex pubescens total flavonoids; BDNF, brain-derived neurotrophic factor; MCAO, middle cerebral artery occlusion; model, MCAO operated without pretreatment; IPTF-1, rats pretreated with $200 \mathrm{mg} / \mathrm{kg}$ IPTF; IPTF-2, rats pretreated with $100 \mathrm{mg} / \mathrm{kg}$ IPTF; IOD, integrated optical density.

IL-10, TNF- $\alpha$ and NO and the activity NOS against ischemic injury.

Effects of IPTF on the expression of BDNF, GDNF and VEGF in the cortex and hippocampus CAl subfields of MCAO rats. The expressions of BDNF, GDNF and VEGF in the cortex and hippocampus CA1 subfields were detected by immunohistochemical staining. Immunohistochemistry demonstrated that IPTF pretreatment significantly increased the expression of BDNF, GDNF and VEGF in cortex and hippocampus CA1 subfields of ischemic hemispheres when compared with the model group $(\mathrm{P}<0.05$ and $\mathrm{P}<0.01$; Figs. $3-5)$.

\section{Discussion}

Mao Dong Qing is derived from the dry roots of Ilex pubescens Hook et Arn. The extracts prepared from the Ilex pubescens roots have a number of biological activities including analgesic, dilating blood vessels, reducing plasma viscosity, inhibiting platelet aggregation, thrombus and enhancing hypoxia-resistance ability (17,31-34). The authors' previous studies (19-21) demonstrated that flavonoids are the prominent active compound in Ilex pubescens and they had neuroprotective effects against cerebral ischemia, which, to the best of our knowledge, has not been reported previously. However, the specific mechanisms underlying the neuroprotective effects of Ilex pubescens remain unknown. In the present study, the potential anti-inflammatory and neuroprotective effects of IPTF were examined.

It was observed that IPTF pretreatment effectively improved neurological deficits, reduced cerebral infarct volume and attenuated brain pathological lesions in a dose-dependent manner in rats with cerebral I/R injury (Figs. 1 and 2; Tables I and II). Therefore, these results observations indicate that IPTF has neuroprotective effects on the cerebral ischemic injury.

The inflammatory response is partly attributable to the pathogenic progression of cerebral ischemic injury, and the associated inflammatory cells and mediators (35). It is well known that cytokines are a group of small glycoproteins $(\sim 25 \mathrm{kDa})$ that serve important roles in the immune response. 

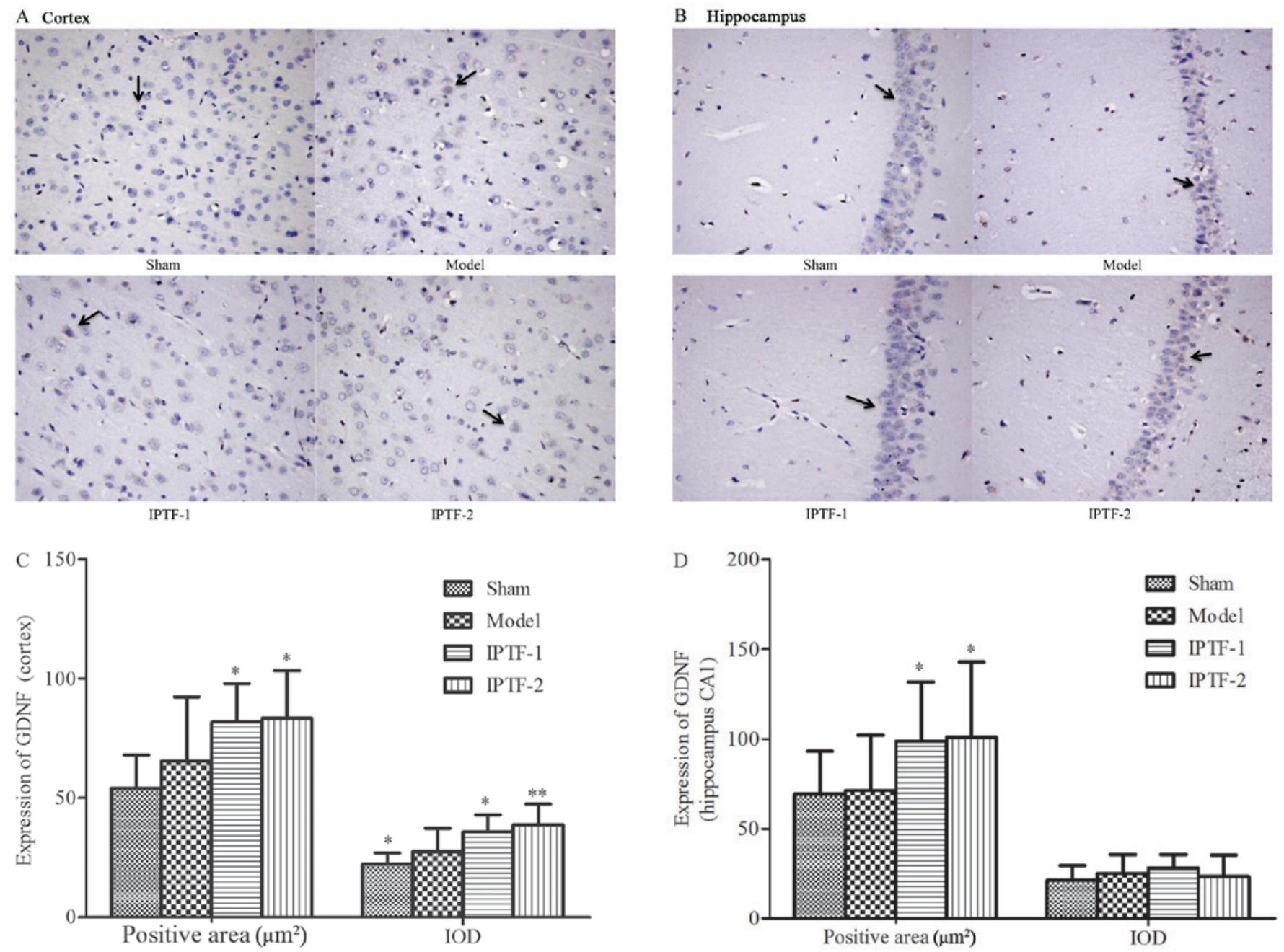

Figure 4. Effect of IPTF on the expression of GDNF in the cortex and hippocampus CA1 subfields of MCAO rats. Protein expression levels of GDNF were detected by immunohistochemical staining in (A) the cortex and (B) the hippocampus CA1 subfields. GDNF is stained in brown and is indicated by arrows; it was primarily localized in the cytoplasm of neurons and gliacytes. Magnification, $\mathrm{x} 400$. GDNF positive immunohistochemical products in the (C) cortex and (D) hippocampus CA1 subfields were counted using Image-Pro Plus 5.1 software, and are expressed as the positive signal area ( $\left.\mu \mathrm{m}^{2}\right)$ and the IOD. Values are expressed as the mean \pm standard deviation ( $\mathrm{n}=6$ random rat brain tissues/group). ${ }^{*} \mathrm{P}<0.05$ and ${ }^{* *} \mathrm{P}<0.01$ vs. model group. IPTF, Ilex pubescens total flavonoids; GDNF, glial cell-derived neurotrophic factor; MCAO, middle cerebral artery occlusion; model, MCAO operated without pretreatment; IPTF-1, rats pretreated with $200 \mathrm{mg} / \mathrm{kg}$ IPTF; IPTF-2, rats pretreated with $100 \mathrm{mg} / \mathrm{kg}$ IPTF; IOD, integrated optical density.

Cytokines are produced in response to a variety of stimuli including pathogens, inflammation or injury, and exert their effects by binding to their receptors. The major cytokines associated with inflammation in cerebral ischemic injury are IL-1 $\beta$, TNF- $\alpha$ and IL-10 (36). Under normal conditions, there is a balance between the pro-inflammatory cytokines IL-1 $\beta$ and TNF- $\alpha$, and the anti-inflammatory cytokine IL-10. Inflammatory cells secrete proinflammatory and anti-inflammatory cytokines during cerebral ischemic injury; their interactions determine the progression of inflammation. When excessive levels of pro-inflammatory cytokines are released as opposed to anti-inflammatory cytokines, inflammation is promoted, thus inducing tissue damage. The levels of IL-1 $\beta$ and TNF- $\alpha$ were upregulated following MCAO or global brain ischemia, however, the level of IL-10 was downregulated (37). A previous study demonstrated that the proinflammatory cytokines IL-1 $\beta$ and TNF- $\alpha$ were important mediators involved in brain injury, and IL-10 was observed to inhibit the production of pre-inflammatory cytokines, thereby exhibiting neuroprotective properties (38). The present study confirmed that
IL-1 $\beta$ and TNF- $\alpha$ were induced by ischemia; IL-1 $\beta$ was downregulated in the brain tissues of the IPTF-1 group $(200 \mathrm{mg} / \mathrm{kg}$ IPTF pretreatment) and TNF- $\alpha$ was downregulated in the brain tissues of both IPTF pretreatment groups (200 and $100 \mathrm{mg} / \mathrm{kg}$; IPTF-1 and -2 , respectively). The level of IL-10 in the brain tissue increased in response to cerebral ischemic injury when comparing the model and sham operation groups, however there was no significant difference, while the levels significantly increased when pretreated with $200 \mathrm{mg} / \mathrm{kg}$ IPTF (IPTF-1 vs. model group).

NO is a pre-inflammatory mediator derived from NOS that is involved in inflammation during cerebral ischemic injury. There are at least two isoforms of NOS: cNOS and iNOS (39-41). The cNOS class is comprised of endothelial constitutive NOS (eNOS), which is present in vascular endothelial cells, and nervous constitutive NOS (nNOS), which is present in the central and peripheral nervous system $(42,43)$. eNOS and nNOS are predominantly expressed in the early phase of the ischemia, whereas iNOS is primarily expressed in the late phase. In the early phase of cerebral ischemia, NO is 

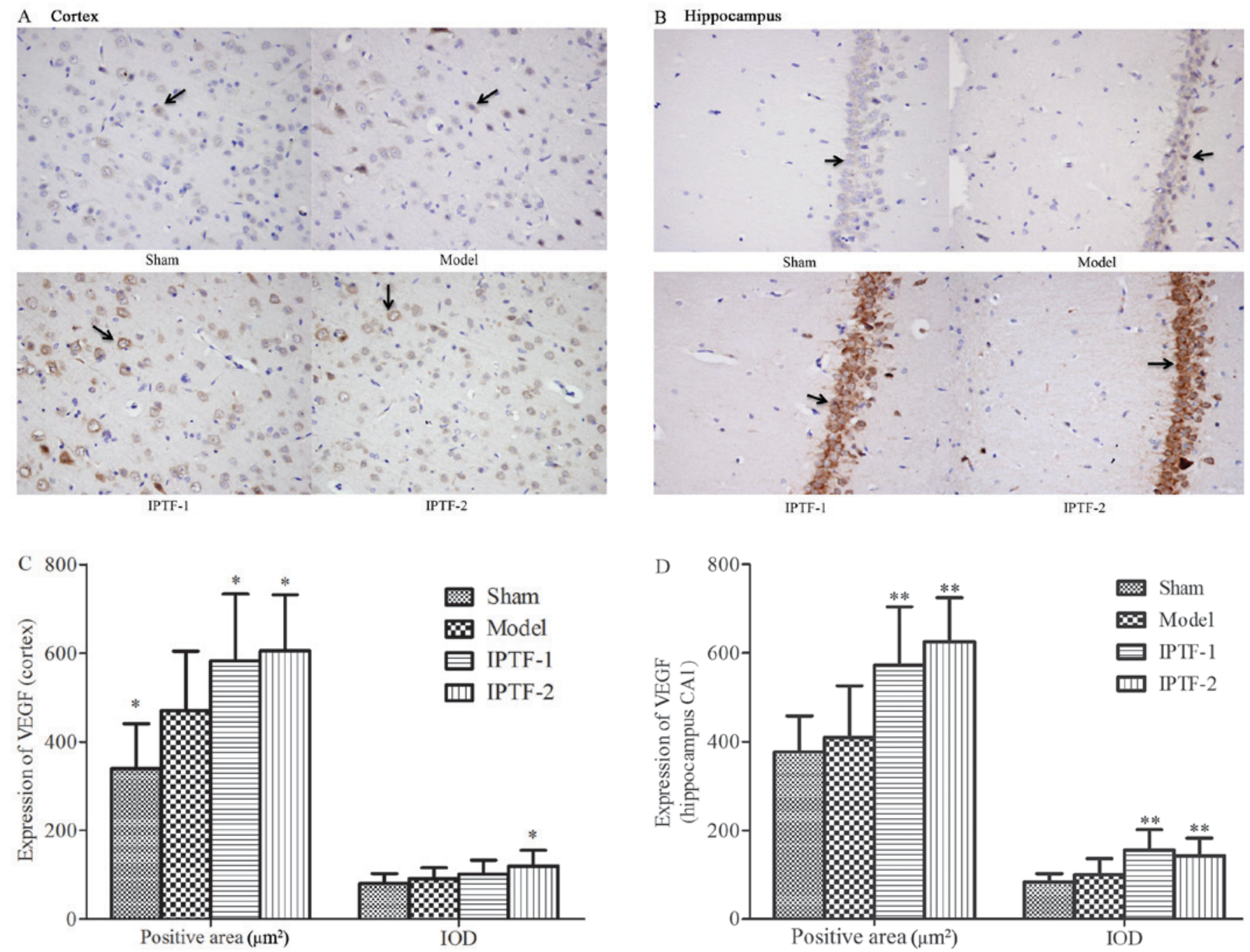

Figure 5. Effect of IPTF on the expression of VEGF in the cortex and hippocampus CA1 subfields of MCAO rats. Protein expression levels of VEGF were detected by immunohistochemical staining in (A) the cortex and (B) the hippocampus CA1 subfields. VEGF is stained in brown and is indicated by arrows; it was primarily localized in the cytoplasm of neurons and vascular endothelial cells. Magnification, $\mathrm{x} 400$. VEGF positive immunohistochemical products in the (C) cortex and (D) hippocampus CA1 subfields were counted using Image-Pro Plus 5.1 software, and are expressed as the positive signal area $\left(\mu \mathrm{m}^{2}\right)$ and the IOD. Values are expressed as the mean \pm standard deviation ( $\mathrm{n}=6$ random rat brain tissues/group). ${ }^{*} \mathrm{P}<0.05$ and ${ }^{* * *} \mathrm{P}<0.01$ vs. model group. IPTF, Ilex pubescens total flavonoids; VEGF, vascular endothelial growth factor; MCAO, middle cerebral artery occlusion; model, MCAO operated without pretreatment; IPTF-1, rats pretreated with $200 \mathrm{mg} / \mathrm{kg}$ IPTF; IPTF-2, rats pretreated with $100 \mathrm{mg} / \mathrm{kg}$ IPTF; IOD, integrated optical density.

predominantly produced by nNOS to cause brain damage, and in the late phase, NO is predominantly produced by iNOS (44). In addition, iNOS mediates cerebral ischemic injury, potentially via the action of $\mathrm{NO}$ on the mitochondrial respiratory chain, resulting in energy depletion (30). A previous study has indicated that the expression levels of iNOS and nNOS increased, however, eNOS expression decreased in MCAO rats (45). A nNOS blocker, 7-NI, has been observed to attenuate the disruption in the blood-brain barrier following transient focal cerebral ischemia; it also improved neurological deficits and reduced the area of cerebral infarction $(46,47)$. In the present study, the level of NO, and the activities of TNOS, iNOS and cNOS were detected $24 \mathrm{~h}$ following the induction of cerebral ischemia. The levels of NO, TNOS, iNOS and cNOS significantly increased in the MCAO model group, which is consistent with the results of previous studies (8-10). The levels of NO, TNOS, iNOS and cNOS significantly decreased in the two IPTF pretreatment groups when compared to the model group. These results indicated that the neuroprotective effects of IPTF might be achieved via a decrease in the levels of inflammation-associated molecules in brain tissues.

Neurotrophins are a family of peptide growth factors that include BDNF, GDNF and VEGF. These growth factors have been demonstrated to protect neurons from damage, and are therefore very important in treatments for neurological disorders including Parkinson's disease, ischemic stroke and Alzheimer's disease $(11,13,48-53)$. Neurotrophins have also been reported to improve angiogenesis, neurogenesis and neurite outgrowth in the brain, and overexpression of these factors has demonstrated their therapeutic actions in cerebral ischemia (54). BDNF was first identified in cerebral ischemia, promoting neurological function recovery and improving neurogenesis (55).

Previous research has demonstrated that the mechanism underlying the neuroprotective effect of GDNF is associated with antiapoptosis and antioxidative effects, and also the induction of progenitor cell proliferation in rats with stroke (56-58). A previous report indicated that BDNF and GDNF may be 
potential therapeutic target proteins for ischemic stroke (59). Therefore, the present study investigated the changes in BDNF and GDNF expression in the cortex and hippocampus CA1 of MCAO rats pretreated with IPTF. The results indicated that the expression of BDNF and GDNF in the cortex and hippocampus CA1 subfields were upregulated in the two IPTF groups following cerebral I/R. Taking the results of the aforementioned previous studies into account, it was proposed that the effect of IPTF on reducing neuronal necrosis may be associated with the upregulation of BDNF and GDNF expression.

A previous study indicated that the expression of VEGF increases following cerebral I/R (60). VEGF is a factor associated with angiogenesis and vascular permeability. Hypoxic conditions can induce the transcriptional and post-transcriptional regulation of VEGF expression in the brain tissue, which is associated with an increase in angiogenesis (61-64). The expression of VEGF is also increased follow cerebral ischemia, which accelerates the formation of novel blood vessels in the brain $(65,66)$. It has been reported that the neuroprotective effects of VEGF are primarily focused on activating the phosphoinositide 3-kinase/protein kinase B pathway, inhibiting the activation of caspase- 3 , the specific potassium currents, the extracellular signal-regulated kinase signaling pathway and the endoplasmic reticulum stress pathway, and increasing the proliferation, migration and differentiation of neuronal progenitors simultaneously (13). In the present study, the expression of VEGF was observed in the hippocampus CA1 and cortex and the findings were consistent with those of previous reports $(67,68)$. The results demonstrated that the expression of VEGF in the hippocampus CA1 and cortex were upregulated in the MCAO model group, particularly in the cortex, which was significantly different to the expression observed in the sham operation group. In addition, pretreatment with IPTF significantly upregulated the expression of VEGF in comparison with the model group. These results indicated that the neuroprotective effects of IPTF may be achieved via the upregulated expression of VEGF in the hippocampus CA1 and cortex.

The recovery of nervous function is a complex process, which is the result of interactions between a number of factors (69-74). The behavioral tests used in the present study were designed to evaluate the level of brain damage in rats, and neurological deficit scores were significantly decreased in rats pretreated with a high dose of IPTF. In addition, IPTF ameliorated the histological injury in brain tissues. In conclusion, the results of the present study suggested that IPTF pretreatment may have neuroprotective effects in cerebral ischemic injury, which may be closely associated with the decreased production of certain proinflammatory cytokines including NO, IL-1 $\beta$ and TNF- $\alpha$, the increased production of the anti-inflammatory cytokine IL-10, the inhibition of TNOS, iNOS and cNOS activities, and the upregulation of BDNF, GDNF and VEGF expression. With further elucidation of its underlying mechanisms of action and clinical verification, IPTF may be a potential neuroprotective treatment for cerebral ischemic injuries.

\section{Acknowledgements}

The present study was supported by the National Natural Science Foundation of China (grant nos. U1204827 and
81503206) and the Fundamental Scientific Research Funds for the Henan Universities (grant no. 2014KYYWF-QN27). The authors would like to thank Professor Chengming Dong and Professor Suiqing Chen (Department of Medicinal Plants, School of Pharmacy, Henan University of Chinese Medicine, Zhengzhou, China) for identifying the plant material.

\section{References}

1. Zhang Y, Zhang S, Li H, Huang M, Xu W, Chu K, Chen L and Chen X: Ameliorative effects of Gualou Guizhi decoction on inflammation in focal cerebral ischemic-reperfusion injury. Mol Med Rep 12: 988-994, 2015.

2. Duong TT, Chami B, McMahon AC, Fong GM, Dennis JM, Freedman SB and Witting PK: Pre-treatment with the synthetic antioxidant T-butyl bisphenol protects cerebral tissues from experimental ischemia reperfusion injury. J Neurochem 130: 733-747, 2014.

3. Liu LP, Xu AD, Wong LK, Wang DZ, Wang YJ and Expert consensus group of the evaluation $\&$ intervention of collateral circulation for ischemic stroke: Chinese consensus statement on the evaluation and intervention of collateral circulation for ischemic stroke. CNS Neurosci Ther 20: 202-208, 2014.

4. Marmagkiolis K, Hakeem A, Cilingiroglu M, Gundogdu B, Iliescu C, Tsitlakidou D and Katramados A: Safety and efficacy of stent retrievers for the management of acute ischemic stroke: Comprehensive review and meta-analysis. JACC Cardiovasc Interv 8: 1758-1765, 2015.

5. Asdaghi $\mathrm{N}$ and Romano JG: Dual antiplatelet therapy in acute ischemic stroke. Curr Atheroscler Rep 17: 37, 2015.

6. Niu PP, Guo ZN, Jin H, Xing YQ and Yang Y: Antiplatelet regimens in the long-term secondary prevention of transient ischaemic attack and ischaemic stroke: An updated network meta-analysis. BMJ Open 6: e009013, 2016.

7. Brouns R and De Deyn PP: The complexity of neurobiological processes in acute ischemic stroke. Clin Neurol Neurosurg 111: 483-495, 2009.

8. Hu XM, Zhou MM, Hu XM and Zeng FD: Neuroprotective effects of scutellarin on rat neuronal damage induced by cerebral ischemia/reperfusion. Acta Pharmacol Sin 26: 1454-1459, 2005.

9. Jung YS, Park JH, Kim H, Kim SY, Hwang JY, Hong KW, Bae SS, Choi BT, Lee SW and Shin HK: Probucol inhibits LPS-induced microglia activation and ameliorates brain ischemic injury in normal and hyperlipidemic mice. Acta Pharmacol Sin 37: 1031-1044, 2016.

10. Zhang F, Li N, Jiang L, Chen L and Huang M: Neuroprotective effects of (-)-epigallocatechin-3-gallate against focal cerebral ischemia/reperfusion injury in rats through attenuation of inflammation. Neurochem Res 40: 1691-1698, 2015.

11. Mattson MP, Maudsley S and Martin B: BDNF and 5-HT: A dynamic duo in age-related neuronal plasticity and neurodegenerative disorders. Trends Neurosci 27: 589-594, 2004.

12. Zhang Y, Lan R, Wang J, Li XY, Zhu DN, Ma YZ, Wu JT and Liu ZH: Acupuncture reduced apoptosis and up-regulated BDNF and GDNF expression in hippocampus following hypoxia-ischemia in neonatal rats. J Ethnopharmacol 172: 124-132, 2015.

13. Yang J, Guo L, Liu R and Liu H: Neuroprotective effects of VEGF administration after focal cerebral ischemia/reperfusion: Dose response and time window. Neurochem Int 60: 592-596, 2012.

14. Miao M, Zhang X and Wang L: Persimmon leaf flavonoid induces brain ischemic tolerance in mice. Neural Regen Res 8: 1376-1382, 2013.

15. Hsu SC and Chung JG: Anticancer potential of emodin. BioMedicine 2: 108-116, 2012.

16. Hao JX and Yang CZ: Research progress on pharmacological action and clinical application of Ilex pubescens. Heilongjiang Med J 23: 592-593, 2010 (In Chinese).

17. Xiong H, Zhao F, Bi J, Zhang Y, Zhao G, Chen X, Li Y, Yan R, Zhao Q, Qiao $\mathrm{H}$ and Zhang G: Two new compounds from the roots of Ilex pubescens and their cytotoxic activity. J Nat Med 70: 673-678, 2016.

18. Zhonghuabencao SAoTCMotPsRoCECo: Zhonghuabencao. Shanghai Science and Technology Press, Shanghai 1997.

19. Zhang F, Zhang XL and Miao MS: Effect of flvonoids of Ilex pubescens on blood stasis with cerebral ischemia. Chin J Exp Tradit Med Form 18: 187-191, 2012 (In Chinese). 
20. Cheng X, Zhang XL and Miao MS: Effect of total flvonoids from radix Ilecis pubescens on rat cerebral ischemia models. Tradit Chin Drug Res Pharmacol 23: 640-643, 2012 (In Chinese).

21. Zhang F, Zhang XL and Miao MS: Effect on total flvonoids of radix Ilicis pubescentis in mice models of cerebral ischemia Tradit Chin Drug Res Pharmacol 23: 409-412, 2012 (In Chinese).

22. Feng SX, Miao MS, Miao JX, Xu P, Shao P and Hou CJ: Simultaneous separation and purification technology of total flavonoids and total saponins from Ilex pubescens by AB-8 macroporous resin. Chin J Exper Trad Med Form: 5-8, 2012

23. Xu YH, Miao MS, Xu P and Feng SX: Determination on content of total flavoniods in the effective fraction of Ilex pubescens Hook. Chin J Chin Med: 826-827, 2011.

24. Koizumi J, Yoshida Y, Nakazawa T and Ooneda G: Experimental studies of ischemic brain edema: I. A new experiment model of cerebral embolism in rats in which recirculation can be introduced in the ischemic area. Jpn J Stroke 8: 1-8, 1986.

25. Nagasawa $\mathrm{H}$ and Kogure K: Correlation between cerebral blood flow and histologic changes in a new rat model of middle cerebral artery occlusion. Stroke 20: 1037-1043, 1989.

26. Longa EZ, Weinstein PR, Carlson S and Cummins R: Reversible middle cerebral artery occlusion without craniectomy in rats. Stroke 20: 84-91, 1989.

27. Belayev L, Alonso OF, Busto R, Zhao W and Ginsberg MD: Middle cerebral artery occlusion in the rat by intraluminal suture. Neurological and pathological evaluation of an improved model. Stroke 27: 1616-1622, 1996. discussion 1623.

28. Yang Y, Shuaib A and Li Q: Quantification of infarct size on focal cerebral ischemia model of rats using a simple and economical method. J Neurosci Methods 84: 9-16, 1998.

29. Kato H, Liu Y, Araki T and Kogure K: Temporal profile of the effects of pretreatment with brief cerebral ischemia on the neuronal damage following secondary ischemic insult in the gerbil: Cumulative damage and protective effects. Brain Res 553: 238-242, 1991.

30. Rodrigo J, Fernández AP, Serrano J, Peinado MA and Martínez A: The role of free radicals in cerebral hypoxia and ischemia. Free Radic Biol Med 39: 26-50, 2005.

31. Wang JR, Zhou H, Jiang ZH, Wong YF and Liu L: In vivo anti-inflammatory and analgesic activities of a purified saponin fraction derived from the root of Ilex pubescens. Biol Pharm Bull 31: 643-650, 2008.

32. Fujimoto T, Fujimura K and Kuramoto A: Electrophysiological evidence that glycoprotein IIb-IIIa complex is involved in calcium channel activation on human platelet plasma membrane. J Biol Chem 266: 16370-16375, 1991.

33. Xing XD, Zhang Q, Feng F and Liu WY: Chemical constituents from stems of Ilex pubescens. Zhong Yao Cai 35: 1429-1431, 2012.

34. Yang ML and Pang PK: The vascular effects of Ilex pubescens. Planta Med 4: 262-265, 1986

35. Muir KW, Tyrrell P, Sattar N and Warburton E: Inflammation and ischaemic stroke. Current opinion in neurology 20: 334-342, 2007.

36. Han HS and Yenari MA: Cellular targets of brain inflammation in stroke. Curr Opin Investig Drugs 4: 522-529, 2003.

37. Hansel G, Tonon AC, Guella FL, Pettenuzzo LF, Duarte T, Duarte MMMF, Oses JP, Achaval M and Souza DO: Guanosine protects against cortical focal ischemia. Mol Neurobiol 52: 1791-1803, 2015

38. Zhu Y, Chen X, Liu Z, Peng YP and Qiu YH: Interleukin-10 protection against lipopolysaccharide-induced neuro-inflammation and neurotoxicity in ventral mesencephalic cultures. Int J Mol Sci 17: pii: E25. 2015.

39. Moncada S, Palmer RM and Higgs EA: Nitric oxide: Physiology, pathophysiology, and pharmacology. Pharmacol Rev 43: 109-142, 1991.

40. Hibbs JB Jr, Taintor RR, Vavrin Z and Rachlin EM: Nitric oxide: A cytotoxic activated macrophage effector molecule. Biochem Biophys Res Commun 157: 87-94, 1988.

41. Förstermann U, Schmidt HH, Pollock JS, Sheng H, Mitchell JA, Warner TD, Nakane M and Murad F: Isoforms of nitric oxide synthase. Characterization and purification from different cell types. Biochem Pharmacol 42: 1849-1857, 1991.

42. Bredt DS and Snyder SH: Isolation of nitric oxide synthase, a calmodulin requiring enzyme. Proc Natl Acad Sci USA 87: 682-685, 1990.

43. Knowles RG, Palacios M, Palmer RM and Moncada S: Kinetic characteristics of nitric oxide synthase from rat brain. Biochem J 269: 207-210, 1990.
44. Hashiguchi A, Yano S, Morioka M, Hamada J, Ushio Y, Takeuchi Y and Fukunaga K: Up-regulation of endothelial nitric oxide synthase via phosphatidylinositol 3-kinase pathway contributes to ischemic tolerance in the CA1 subfield of gerbil hippocampus. J Cereb Blood Flow Metab 24: 271-279, 2004.

45. Koh PO: Ferulic acid modulates nitric oxide synthase expression in focal cerebral ischemia. Lab Anim Res 28: 273-278, 2012.

46. Jiang Z, Li C, Arrick DM, Yang S, Baluna AE and Sun H: Role of nitric oxide synthases in early blood-brain barrier disruption following transient focal cerebral ischemia. PLoS One 9: e93134, 2014.

47. Iadecola C, Zhang F, Casey R, Nagayama M and Ross ME: Delayed reduction of ischemic brain injury and neurological deficits in mice lacking the inducible nitric oxide synthase gene. J Neurosci 17: 9157-9164, 1997.

48. Kitagawa H, Hayashi T, Mitsumoto Y, Koga N, Itoyama Y and Abe K: Reduction of ischemic brain injury by topical application of glial cell line-derived neurotrophic factor after permanent middle cerebral artery occlusion in rats. Stroke 29: 1417-1422, 1998.

49. Sheikh MA, Malik YS, Xing Z, Guo Z, Tian H, Zhu X and Chen X: Polylysine-modified polyethylenimine (PEI-PLL) mediated VEGF gene delivery protects dopaminergic neurons in cell culture and in rat models of Parkinson's Disease (PD). Acta Biomater 54: 58-68, 2017.

50. Sarkar S, Raymick J and Imam S: Neuroprotective and therapeutic strategies against parkinson's disease: Recent perspectives. Int J Mol Sci 17: pii: E904, 2016.

51. Echeverria V, Barreto GE, Ávila-Rodriguez M, Tarasov VV and Aliev G: Is VEGF a key target of cotinine and other potential therapies against Alzheimer disease? Curr Alzheimer Res: Mar 29, 2017 (Epub ahead of print).

52. Nigam SM, Xu S, Kritikou JS, Marosi K, Brodin L and Mattson MP: Exercise and BDNF reduce A $\beta$ production by enhancing $\alpha$-secretase processing of APP. J Neurochem 142: 286-296, 2017.

53. Wei S: Potential therapeutic action of natural products from traditional Chinese medicine on Alzheimer's disease animal models targeting neurotrophic factors. Fundam Clin Pharmacol 30: 490-501, 2016

54. Shimamura M, Sato N and Morishita R: Experimental and clinical application of plasmid DNA in the field of central nervous diseases. Curr Gene Ther 11: 491-500, 2011.

55. Choi Y, Kang SG and Kam KY: Changes in the BDNFimmunopositive cell population of neocortical layers I and II/III after focal cerebral ischemia in rats. Brain Res 1605: 76-82, 2015.

56. Nicole O, Ali C, Docagne F, Plawinski L, MacKenzie ET, Vivien D and Buisson A: Neuroprotection mediated by glial cell line-derived neurotrophic factor: Involvement of a reduction of NMDA-induced calcium influx by the mitogen-activated protein kinase pathway. J Neurosci 21: 3024-3033, 2001.

57. Deng HL and Zhang JT: Anti-lipid peroxidative effect of ginsenoside Rb1 and Rg1. Chin Med J (Engl) 104: 395-398, 1991.

58. Kobayashi T, Ahlenius H, Thored P, Kobayashi R, Kokaia Z and Lindvall O: Intracerebral infusion of glial cell line-derived neurotrophic factor promotes striatal neurogenesis after stroke in adult rats. Stroke 37: 2361-2367, 2006.

59. Liu Y, Wang S, Luo S, Li Z, Liang F, Zhu Y, Pei Z and Huang R: Intravenous PEP-1-GDNF is protective after focal cerebral ischemia in rats. Neurosci Lett 617: 150-155, 2016.

60. Lin TN, Te J, Lee M, Sun GY and Hsu CY: Induction of basic fibroblast growth factor (bFGF) expression following focal cerebral ischemia. Brain Res Mol Brain Res 49: 255-265, 1997.

61. Raber J, Fan Y, Matsumori Y, Liu Z, Weinstein PR, Fike JR and Liu J: Irradiation attenuates neurogenesis and exacerbates ischemia-induced deficits. Ann Neurol 55: 381-389, 2004.

62. Huang Z, Huang PL, Ma J, Meng W, Ayata C, Fishman MC and Moskowitz MA: Enlarged infarcts in endothelial nitric oxide synthase knockout mice are attenuated by nitro-l-arginine. J Cereb Blood Flow Metab 16: 981-987, 1996.

63. Nagayama M, Aber T, Nagayama T, Ross ME and Iadecola $C$ : Age-dependent increase in ischemic brain injury in wild-type mice and in mice lacking the inducible nitric oxide synthase gene. J Cereb Blood Flow Metab 19: 661-666, 1999.

64. Zhu DY, Liu SH, Sun HS and Lu YM: Expression of inducible nitric oxide synthase after focal cerebral ischemia stimulates neurogenesis in the adult rodent dentate gyrus. J Neurosci 23: 223-229, 2003. 
65. Zhang ZG, Zhang L, Tsang W, Soltanian-Zadeh H, Morris D, Zhang R, Goussev A, Powers C, Yeich T and Chopp M: Correlation of VEGF and angiopoietin expression with disruption of blood-brain barrier and angiogenesis after focal cerebral ischemia. J Cereb Blood Flow Metab 22: 379-392, 2002.

66. Ogunshola OO, Stewart WB, Mihalcik V, Solli T, Madri JA and Ment LR: Neuronal VEGF expression correlates with angiogenesis in postnatal developing rat brain. Brain Res Dev Brain Res 119: 139-153, 2000

67. Krum JM, Mani N and Rosenstein JM: Angiogenic and astroglial responses to vascular endothelial growth factor administration in adult rat brain. Neuroscience 110: 589-604, 2002.

68. Zhang ZG, Zhang L, Jiang Q, Zhang R, Davies K, Powers C, Bruggen Nv and Chopp M: VEGF enhances angiogenesis and promotes blood-brain barrier leakage in the ischemic brain. J Clin Invest. 106: 829-838, 2000.

69. Ishii T, Ueyama T, Shigyo M, Kohta M, Kondoh T, Kuboyama T, Uebi T, Hamada T, Gutmann DH, Aiba A, et al: A novel Rac1-GSPT1 signaling pathway controls astrogliosis following central nervous system injury. J Biol Chem 292: 1240-1250, 2017.
70. Sulaiman WA: Transforming growth factor- $\beta$ promotes axonal regeneration after chronic nerve injury. Spine (Phila Pa 1976) 41 (Suppl 7): S29, 2016.

71. Lin Q, Wong HL, Tian FR, Huang YD, Xu J, Yang JJ, Chen PP, Fan ZL, Lu CT and Zhao YZ: Enhanced neuroprotection with decellularized brain extracellular matrix containing bFGF after intracerebral transplantation in Parkinson's disease rat model. Int J Pharm 517: 383-394, 2017.

72. Lei C, Wu B, Cao T, Liu M and Hao Z: Brain recovery mediated by toll-like receptor 4 in rats after intracerebral hemorrhage. Brain Res 1632: 1-8, 2016.

73. Furukawa S: Basic research on neurotrophic factors and its application to medical uses. Yakugaku Zasshi 135: 1213-1226, 2015 (In Japanese)

74. Stetler RA, Gao Y, Leak RK, Weng Z, Shi Y, Zhang L, Pu H, Zhang F, Hu X, Hassan S, et al: APE1/Ref-1 facilitates recovery of gray and white matter and neurological function after mild stroke injury. Proc Natl Acad Sci USA 113: E3558-E3567, 2016. 